\title{
Agustín de Hipona en la obra arendtiana temprana
}

\author{
Agostinho de Hipona nas primeiras leituras arendtianas
}

Augustine of Hippo in the early Arendtian reading

DOI: $10.20873 / \mathrm{rpv} 6 \mathrm{n} 2-06$

\author{
Ari Angelina Costamagna Fernández \\ Orcid: https://orcid.org/0000-0001-5306-6474 \\ Email: ari.costamagna.fernandez@mi.unc.edu.ar
}

\begin{abstract}
Resumen
El presente artículo se enmarca en una investigación en proceso, en la cual exploramos la lectura arendtiana de Agustín de Hipona, a partir de la obra temprana, El concepto de amor en San Agustín (2001). Consideramos que la investigación de la disertación doctoral, obra tan poco explorada por sus intérpretes más relevantes, puede brindarnos nuevas claves de lectura de la teoría política de Hannah Arendt, complejizando nuestro acercamiento a su lectura heterodoxa de la tradición de pensamiento filosófico. Según nuestra hipótesis es en la tesis doctoral de 1929, donde comienza a despuntar la dimensión política del problema en sentido amplio, a partir de la pregunta sobre lo específicamente humano, siempre sujeto a la tensión constante entre un "ya no" y un "todavía no" (Arendt, 2001, p. 311; Agustín, 2005, p. XI, 21,27). Sólo en obras posteriores la lectura de la fuente agustiniana -enfocada cada vez más en las Confesiones (2005) y en La Ciudad de Dios (1958) queda explícitamente articulada con la elaboración de la trama más conocida de la fenomenología de la acción política; en la que numerosos y destacados intérpretes han situado el giro hacia la política de la alumna de Heidegger y Jaspers (Young Bruehl, 2006; Canovan, 2002; Benhabib, 2000; Forti, 2001). Si bien estas autoras reconocen la influencia de la formación temprana de Arendt, en general todas acentúan la ruptura con todo su ámbito de formación universitaria en filosofía y teología.
\end{abstract}

\section{Palabras clave}

Hannah Arendt; Formación temprana; Agustín de Hipona.

\section{Abstract}

This article is a part of an ongoing investigation, where we explore Arendt's reading of Augustine of Hippo, starting fron the early work, El concepto de amor en San Agustín (2001). We consider that the research of the 
doctoral dissertation, a work so little explored by her most relevant exponent, can provide us new keys to reading Hannah Arendt's political theory, making our approach more complex to her heterodox reading of the tradition of philosophical thought. According to our hypothesis, is in the 1929 doctoral thesis, where the political dimension of the problem in a broad sense begins to emerge, from the question of what is specifically human, always subject to the constant tension between a "no longer" and a "no yet" (Arendt, 2001, p. 311; Agustín, 2005, p. XI, 21,27). Only in later works is the reading of the Augustinian source - increasingly focused on the Confesiones (2005) and La Ciudad de Dios (1958) - is explicitly articulated with the elaboration of the most well-known plot of the phenomenology of political action; in which numerous and prominent exponents have located Heidegger and Jaspers' student, her turn towards the politics (Young Bruehl, 2006; Canovan, 2002; Benhabib, 2000; Forti, 2001). While these authors acknowledge the influence of Arendt's early education, in general they all accentuate her break with the entire scope of her university education in philosophy and theology.

\section{Keywords}

Hannah Arendt. Early education. Augustine of Hippo.

\section{Resumo}

Este artigo faz parte de uma investigação em andamento, na qual exploramos a leitura arendtiana de Agostinho de Hipona, partindo da obra inicial, El concepto de amor en San Agustín (2001). Consideramos que a investigação da dissertação doutoral, obra tão pouco explorada pelos seus mais relevantes intérpretes, pode abrir novas chaves de leitura da teoria política de Hannah Arendt, tornando mais complexo nossa abordagem na sua leitura heterodoxa da tradição do pensamento filosófico. Segundo nossa hipótese é a tese doutoral de 1929, na qual a dimensão política do problema em um sentido amplo começa a emergir, a partir da questão do que é especificamente humano, sempre sujeito à constante tensão entre um "não mais" e um "ainda não" (Arendt, 2001, p. 311; Agustín, 2005, p. XI, 21,27). Somente em posteriores trabalhos a leitura da fonte agostiniana, focada cada vez mais nas Confesiones (2005) y na Ciudad de Dios (1958) fica articulada explicitamente com a elaboração da trama mais conhecida da fenomenologia da ação política; na qual numerosos e importantes intérpretes têm situado o redirecionamento para a política da aluna do Heidegger e Jaspers (Young Bruehl, 2006; Canovan, 2002; Benhabib, 2000; Forti, 2001). Embora essas autoras reconheçam a influência na formação inicial de Arendt, em geral, todas elas acentuam a ruptura com o seu âmbito de formação universitária em filosofia e teologia.

\section{Palavras-chave}

Hannah Arendt; Educação inicial; Agostinho de Hipona.

\section{Introducción}

La heterodoxia de las lecturas de Hannah Arendt nos plantea determinados inconvenientes a la hora de dar con el "pensamiento arendtiano". De hecho, las interpretaciones canónicas y exegéticas remarcan esta dificultad, pero no por ello dejan de insistir en la originalidad y 
la calidad de los problemas que la autora ilumina. La lucidez de la lectura de la tradición de pensamiento filosófico que propone Arendt, a la luz de un diálogo complejo con la historia, con la literatura, con la filosofía, permite pensar su metodología como un tipo de indagación histórico- crítica. En este sentido, Arendt reniega de las lecturas que intentan sintetizar el pensamiento de los autores de la tradición, haciéndolos parte de un canon preestablecido, sin complejizar ni entender las limitaciones y las potencias que aquellos planteos problemáticos abren. Esto es, la lectura arendtiana se establece como una inquisición precisa para "dejar que las contradicciones se alcen tal como son" y así "hacerlas comprensibles como contradicciones y captar qué es lo que ocultan" (ARENDT, 2001, p. 20); no se trata de negar las tensiones e incongruencias, ni de la filosofía ni de la realidad política, en una suerte de idealismo tranquilizador, sino de reconocerlas y de indagar lo que se oculta detrás de ellas:

Estas contradicciones tan fundamentales y flagrantes pocas veces se presentan en autores de segunda línea, en quienes pueden descontarse. En los grandes autores nos llevan hasta el centro mismo de sus obras y son la clave más importante para llegar a la verdadera comprensión de sus problemas y sus nuevos criterios. (...) Es como si Marx, casi al modo de Kierkeegaard y de Nietzsche, mientras usa las herramientas conceptuales de la tradición, trata desesperadamente de pensar en contra de ellas (ARENDT, 2016a, p. 44).

Es precisamente en ese movimiento que Arendt detecta en "los grandes autores", que consiste en pensar con y más allá de la tradición, donde identificamos algunos rasgos de su "metodología” de análisis: ella misma piensa con, más allá de la tradición. Aquí pretendemos explorar estos vaivenes del pensamiento de Hannah Arendt tomando en consideración su primer trabajo de largo aliento, El concepto de amor en San Agustín (2001); una obra de difícil acceso aún para los versados sobre el tema. Aquella oscilación toma consistencia en la brecha entre pasado y futuro como un abismo temporal. Este abismo temporal no es una simple construcción teórica, sino que prefigura y describe la misma brecha temporal abierta en la historia como una herida, vinculada a los sucesos de la década del 20’ y los años que le siguieron ligada a los acontecimientos terribles de la Segunda Guerra Mundial. Aquel abismo se instala en la tensión entre un testamento del que "ya no" podíamos hacer uso, puesto que no podíamos confiar más en las respuestas dadas del pasado, pero "todavía no" estábamos en condiciones de dar 
nuevas respuestas a acontecimientos que dislocaban nuestras categorías de pensamiento dejándolas obsoletas.

Aquí realizaremos nuestros primeros acercamientos a su disertación doctoral, una obra compleja y desprovista de estudios sistemáticos. Consideramos que la falta de interés de los intérpretes más importantes de la obra de Arendt se debe, como muchos han mencionado, a que esta obra tiene poco o nada que ver con su giro hacia la política: más bien se trata de una investigación de corte estrictamente filosófico, atada fuertemente a las influencias de sus maestros de juventud, Heidegger y Jaspers.

No es nuestra intención desconocer los desplazamientos que se produjeron a lo largo de la evolución de la obra arendtiana, ni tampoco obviar el contexto de producción ni las periodizaciones analíticas que suelen hacerse a fin de comprender mejor la obra, pero sí prestar atención a esta obra descuidada y restituir su importancia en el marco de la génesis del pensamiento arendtiano. La hipótesis que guiará nuestra interpretación es que la disertación no implica una ruptura radical con la obra posterior, particularmente con lo que se ha señalado como su giro hacia la política durante el período en Estados Unidos, sino que hay importantes continuidades respecto de la lectura de San Agustín, particularmente de algunos conceptos y tópicos relevantes de su pensamiento.

De este modo, este breve escrito pretende aportar al campo de los estudios arendtianos ofreciendo en primera instancia una reconstrucción del contexto de producción histórico-intelectual en el momento en el que Arendt escribe dicha tesis doctoral. Para realizar esta reconstrucción tomaremos sobre todo la obra de la historiadora Keedus (2015) y sus fuentes. En segunda instancia, brindaremos un enfoque general de lectura, que nos permita resaltar los puntos de partida más relevantes de la obra que analizamos, los cuales a nuestro entender son: por un lado la relevancia de la perspectiva existencialista, sobre todo su diálogo crítico con su maestro de juventud, Heidegger. Allí, tomamos como punto de partida la investigación de Espósito (2009) sobre la lectura heideggeriana de Agustín de Hipona, resaltando su relevancia en el primer período del pensador alemán. Por otro lado, retomaremos la lectura arendtiana de la tradición, cuyo abordaje original ya se encuentra en ciernes en esta obra de juventud, como 
veremos. Dividiremos el escrito en tres partes. En el primero, reconstruiremos el contexto de producción; en el segundo brindaremos el enfoque general de lectura descrito sucintamente arriba. Finalmente, abordaremos brevemente los rasgos de su compleja lectura de la tradición, ya en ciernes en esta obra de juventud.

\section{Contexto histórico-intelectual}

¿Por qué Agustín y el amor? Pensador cristiano y romano, filósofo ligado a la tradición neoplatónica griega, nacido en Tagaste, pequeña ciudad costera del Norte de África. La imagen de Agustín como aquel obispo fundador de la iglesia católica se disuelve lentamente al observar las inflexiones de su historia y de su contexto. Como menciona Arendt en la introducción de su tesis doctoral, si bien su fe cristiana no dejó de crecer en él con el pasar de los años, y como puede verse también en su producción intelectual, los cursos diversos de su pensamiento no pueden simplemente ser reducidos a uno predominante. Muchos pueden ser los ángulos a través de los cuales leer los rasgos "principales" de su obra. En las articulaciones de estas líneas que parecen discurrir en paralelo es donde encontramos la riqueza de sus reflexiones y su actualidad.

Estos cursos de pensamiento que discurren como paralelos pueden leerse de diferentes maneras y aplicarse a determinados conceptos de la filosofía agustiniana, pero lo que aquí nos interesa es la articulación de estos contextos conceptuales a la luz de la lectura arendtiana de Agustín en esta obra de juventud. El análisis propuesto de la joven autora hace aflorar las tensiones que se expresan como contradicciones e incongruencias en el pensamiento del hiponense. Como destaca Jaspers en el dictamen de la disertación, el interés fundamental de Arendt en su trabajo es un objetivo que nunca explicita: se trata de la indagación existencial en el horizonte de la filosofía del obispo. Arendt no busca darle una unidad a un pensamiento que ha perdido su sentido como un todo, intenta buscar la posibilidad de su actualización en sus grietas. La filosofía de Agustín ha perdido su sentido como un todo pero no el sentido del pasado 
que recupera. Así, la lectura arendtiana se detiene en las grietas, las considera como tensiones productivas y en ese sentido las preguntas se formulan desde un horizonte histórico- crítico.

Aquí queremos abordar brevemente el contexto de producción de esta obra de juventud de Hannah Arendt a la luz y al calor de las discusiones de su época, elementos que luego nos ayudarán a pensar los contextos conceptuales que allí la autora diferencia en el horizonte de comprensión de la disertación. En esta línea tomaremos la hipótesis de Keedus (2015), según la cual los debates filosóficos de Weimar condicionaron la formación académica de Hannah Arendt, en virtud de las polémicas antihistoricistas. Las lecturas particulares que se abren allí para la joven estudiante se vinculan con una comprensión particular de la temporalidad, que sugieren nuevas formas de leer el presente a través del pasado y sus fuentes ${ }^{1}$.

En la década de 1920, tal como destaca Keedus, el joven teólogo Friedrich Gogarten escribe un breve ensayo titulado Zwischen den Zeiten (Between the times). Lo que expresa allí es una crítica radical a los lineamientos epistémicos y hermenéuticos dominantes en las humanidades y las ciencias sociales en su época, como menciona Keedus; en esa línea construye una lectura que se caracteriza por un antihistoricismo radical2:

Gogarten formuló su antihistoricismo en términos de antagonismo existencial radical. En términos dramáticos, afirmó el fin de todo un "mundo de ayer", que para él no implicaba nada que valiera la pena defender (KEEDUS, 2015, p. 15, nuestra traducción)33.

\footnotetext{
${ }^{1}$ Sobre la investigación de Keedus mencionaremos dos cosas: por un lado, entendemos que hace un gran aporte sobre el contexto de producción de esta obra, ya que contamos con escasos estudios biográficos sobre este período de juventud. Si bien la biografía de Young-Bruehl se convirtió en una obra canónica en el marco de los estudios arendtianos no le presta la suficiente atención a aquél período de juventud. De todos modos, contrastaremos estas dos lecturas a lo largo de este apartado. Por otro lado, utilizaremos la lectura de Keedus (2015) solo para complejizar este corto período de juventud ya que consideramos que algunas hipótesis son demasiado arriesgadas, sobre todo las proponen una lectura particular de los períodos posteriores. Lo que queremos rescatar de su propuesta de lectura en The crisis of German Historicism: The early political thought of Hannah Arendt and Leo Strauss es que considera a Arendt y a Strauss como hijos de un linaje particular en el sentido de tradición de pensamiento asociado a "la abundancia de discusiones en torno al concepto de "tradición" en sí" (KEEDUS, 2015, p. 14).

${ }^{2}$ Aquellas críticas se alzaban principalmente contra lo que se llamó "teología liberal".

3 "Gogarten formulated his antihistoricism in terms of radical, existential antagonism. In sweeping terms he claimed the end of an entire "world of yesterday," that for him did not involve anything worth upholding" (KEEDUS, 2015, p. 15).
} 
Como menciona Keedus, aunque el historicismo llevaba al menos un siglo de discusiones, a principios del siglo XX comenzó a usarse como "anti-término". Para la intérprete el historicismo como anti-término puede leerse en dos sentidos: por un lado, referido a los modos de relacionarnos con nuestro pasado. En ese sentido para sus críticos,

...significaba, ...escribir "historia simplemente por el bien de historia." Significaba ofrecer estudios "minuciosos" del pasado que eran incapaces de relacionarse de manera significativa con el presente. La aclamada acumulación de materiales y hechos sin valor del historicismo se convirtió para sus críticos en el contrabando de "positivismo" y de los "anticuarios" de las ciencias humanas (KEEDUS, 2015, p. 15, nuestra traducción)4.

Por otro lado, puede usarse en relación a sus "fundamentos filosóficos y normativos", es decir teniendo en cuenta en última instancia las consecuencias ético-filosóficas: "la aproximación historicista se niega a distinguir entre lo correcto o incorrecto, en una versión; en otra, quiere distinguirlos pero no puede" (KEEDUS, 2015, p. 16, nuestra traducción). Por último, la relación que el historicismo encarnaba con sus fuentes impedía pensarlas en su actualidad: “Al cortar sus lazos con el presente, al negarse a juzgar al pasado, de hecho silenciaron las fuentes" (KEEDUS, 2015, p. 16) ${ }^{5}$, intentando cumplir el dictum rankeano: leer el pasado "como realmente fue".

A partir del enfoque radical antihistoricista que propulsó Gogarten, se nucleó un grupo de jóvenes teólogos. Para estos jóvenes investigadores, “stand between the times" significó la posibilidad de acceder a un horizonte de interrogación nuevo en las ciencias humanas. Karl Barth, uno de sus estudiantes y escritor de Die Römerbriefe (1919), en la misma línea interpreta los escritos de Pablo. Como advierte Keedus, y en la voz de Barth: "Pablo se dirigió a sus contemporáneos, como hijo de su época. Sin embargo, es más importante que, como profeta y apóstol del Reino de Dios, verdaderamente le habló a todos los seres humanos de todos los tiempos"

\footnotetext{
4 "For its critics, it meant, first, to write "history merely for the sake of history." It meant to offer "minute" studies of the past that were unable to meaningfully relate to the present. Historicism's acclaimed value-free accumulation of materials and facts became for its critics the smuggling of "positivism" and "antiquarianism" into the human sciences" (Keedus, 2015, p. 15).

5 "By cutting off its bonds to the present, by refusing to judge the past it had in fact silenced the sources" (Keedus, 2015, p. 16).
} 
(BARTH, 1933:1, nuestra traducción) ${ }^{6}$. De este modo, podemos decir que la preocupación hermenéutica de aquellos teólogos radicaba en problematizar el "entre" de nuestro tiempo y el pasado, en donde el muro temporal se vuelve transparente ${ }^{7}$.

En ese marco, nuestra intención es revisar y revisitar bajo otra óptica la centralidad de la influencia heideggeriana en este período de juventud, ligándolo al contexto del producción y discusión intelectual de aquellos años. No negamos que el pensamiento de su joven maestro tenga relevancia en la gestación de sus ideas, sino que consideramos que las lecturas que ponen en evidencia sólo aquella relación deja de lado el contexto histórico en el cual se ubicaban los debates intelectuales de esta época, y sesgan la riqueza de la multifacética lectura arendtiana, que puede entenderse en el marco de un diálogo complejo, interdisciplinario, con las fuentes de la tradición de pensamiento filosófico, así como con las fuentes del pensamiento teológico. En ese sentido, la perspectiva heideggeriana también puede entenderse en un diálogo fluido con aquellos debates.

A propósito del excelente trabajo biográfico de Young Bruehl, la intérprete destaca que en 1924, año en que ingresa a la Universidad de Marburgo, Hannah Arendt fue parte de una intensa revolución apolítica de la que de la mano de Heidegger, la cual tendría peso en su evolución personal e intelectual (YOUNG-BRUEHL, 2006, p. 108). Tal como recuerda luego Arendt y remarcan otros intérpretes, la filosofía académica de los años 20 estaba anquilosada, y ciertos espacios académicos conservadores defendían los ismos que construían la tradición en sentido fuerte (ARENDT, 1971, p. 294; SCOTT \& STARK, 1996, p. 117):

Comúnmente la Universidad les ofrecía [a los estudiantes] las escuelas- los neokantianos, los neohegelianos, los neoplatonistas, etc.- o la vieja disciplina académica, en la cual la filosofía, cuidadosamente dividida en sus campos específicos- epistemología, estética, ética, lógica, y cosas por el estilo-

\footnotetext{
6 "Paul, as a child of his age, addressed his contemporaries. It is, however, far more important that, as Prophet and Apostle of the Kingdom of God, he veritably speaks to all men of every age" (BARTH, 1933, p. 1).

70 como menciona Keedus, “...Barth set out to inquire into "what is there", until the "Wall” between our time and Paul's becomes "transparent" (KEEDUS, 2015, p. 19).
} 
no se transmitía tanto como se ahogaba en un océano de aburrimiento (ARENDT, 1971, p. 294, nuestra traducción) $)^{8}$.

En ese marco, los seminarios de Heidegger le daban vida a la filosofía avejentada y académica; como mencionó luego la autora de Los orígenes: "Thinking has become to life again" (ARENDT, 1971, p. 295).

La biógrafa de Arendt, interpreta la relación con su maestro Martin Heidegger como nodal para comprender este período de juventud. De todos modos, lo que señala sobre todo aquí es el vínculo desde un punto de vista estrictamente personal e incluso, sentimental, ligado a la aventura de juventud entre ambos, que corren el eje de discusión que aquí queremos resaltar. Como dice Young-Bruehl:

...ella se refería a su primer encuentro con la filosofía, en Marburgo, como a la época de su "primer amor". La filosofía fue, en efecto, su primer amor; pero la filosofía encarnada en la persona de Martín Heidegger (YOUNG- BRUEHL, 2006, p. 109).

Heidegger ingresa como catedrático titular a la Universidad de Marburgo el mismo año que Arendt comienza a cursar, 1924, y durante aquel año trabajó arduamente sobre su primer borrador de lo que se convirtió posteriormente Ser y tiempo ${ }^{9}$. En ese sentido, Arendt y los estudiantes que cursaron en ese momento los seminarios impartidos por Heidegger, tuvieron la fortuna de estudiar con uno de los grandes filósofos del siglo XX presenciando la gestación de su obra maestra de juventud. En Marburgo, Heidegger consolidó su posición como catedrático en parte gracias a la influencia de Paul Natorp, tal como menciona la biógrafa; también mantuvo un estrecho vínculo tanto con Bultmann, teórico ligado a la "teología existencialista”, como con el filósofo Nikolai Hartmann, con quienes luego Hannah Arendt cursaría distintos seminarios.

\footnotetext{
8 "The university commonly offered them either the schools - the neo-Kantians, the Neo-Hegelians, the Neo-Platonists, etc.- or the old academic discipline, in which philosophy, neatly divided into its special fields - epistemology, aesthetics, ethics, logic, and the like-was not so much communicated as drowned in an ocean of boredom" (ARENDT, 1971, p. 194).

${ }^{9}$ En el semestre de verano de 1923, Heidegger impartió en la Universidad de Friburgo un seminario que se tituló Ontologie (Hermeneutik der Faktizitä) (Ontología: Hermenéutica de la Facticidad). Este será el primer borrador de Ser y tiempo, sobre el cual siguió trabajando los años sucesivos en la Universidad de Marburgo. No tenemos en claro cuáles seminarios cursó Arendt en este momento.
} 
De todos modos, aunque en este prestigioso estudio, Hannah Arendt: for love of the world (1993), la biógrafa reconstruye acertadamente el contexto de producción que intentamos traer aquí, sus hipótesis acerca de la juventud naif y enamoradiza de la autora de La Condición Humana son un poco apresuradas, y cierran el vínculo intelectual de Arendt con su maestro a su aventura amorosa y no le presta demasiada atención a las controversias intelectuales de la época. Sin lugar a dudas, las enseñanzas de Heidegger marcaron su formación pero no solamente el modo en que describe la biógrafa en la primera parte de la biografía.

En este sentido planteamos que el repertorio de docentes universitarixs con quienes cursó fue mucho más amplio y que tuvieron relevancia en aquel horizonte. En primera instancia, cursa en Berlín un seminario sobre Kierkegaard con un importante erudito católico, Guardini. Por otro lado, hay que reparar que la Universidad de Marburgo fue la casa de estudios de numerosos intelectuales interesados por la teología crítica (o dialéctica), dentro de los cuales encontramos a Rudolf Otto y a Rudolf Bultmann, entre otros. Con este último Arendt cursó un seminario sobre el Nuevo Testamento, enfocado desde la perspectiva antropológica de Pablo. Bultmann también formó parte del círculo Zwischen den Zeit, pero a diferencia de Barth, consideraba que los textos bíblicos no nos hablaban inmediatamente ni a nuestros contemporáneos, sino que la tarea principal de la teología era traducir las Santas Escrituras a los términos y al lenguaje de los cristianos contemporáneos (KEEDUS, 2015, p. 50). Aquellos teólogos heterodoxos convidaban nuevas formas de acercarse a la teología y a la hermenéutica, y abrían el campo de estudios a una concepción "interdisciplinaria”. Por otro lado, Bultmann puso un gran empeño en construir su propuesta teológica en términos existencialistas, intentando desmitologizar (Entmythologisierung ${ }^{10}$ las fuentes bíblicas. Como ya mencionamos, en Marburgo fue colega y amigo de Heidegger, quien también se vio sumido en los debates teológicos de su entorno intelectual.

\footnotetext{
${ }^{10}$ Desmitologización es un término técnico utilizado por Bultmann que alude a (en sus propias palabras) "to arrive at a genuine comprehension of past existential insights, a comprehension that would go beyond the horizons of objectified thinking" (KEEDUS, 2015, p. 50). Keedus toma esta cita de un libro que Bultmann escribe junto a Jaspers. Ver: Karl Jaspers and Rudolf Bultmann (2005): Myth and Christianity: An Inquiry into the Possibility of Religion without Myth. New York: Prometheus Books. Es interesante el vínculo entre Jaspers y Bultmann por sí mismo, pero
} 
Arendt finaliza sus estudios universitarios en la Universidad de Heidelberg. En aquella universidad uno de sus profesores fue Martin Dibelius, quien impulsó nuevas lecturas en el campo de los estudios exegéticos y al interior del grupo Zwischen den Zeiten. Como menciona Keedus, Dibelius criticó las lecturas propuestas por Barth y por Bultmann: al primero le reprochó que apelase a recuperar la voz de Pablo sin atender al contexto histórico, o considerar las circunstancias bajo las cuales sucedieron los hechos, o problematizar las fuentes. Leer a Pablo como pretendía Barth, como si fuese un contemporáneo, carecía de sentido para Dibelius. Contra Bultmann, sostuvo que:

...la comprensión de las figuras bíblicas y su mensaje no puede basarse en una construcción psicológico- sustancialista de sus mentes y personalidades, sino sólo en un análisis histórico-estilístico de la "vida e intereses de las primeras comunidades cristianas" (KEEDUS, 2015, p. 51, nuestra traducción) ${ }^{11}$.

En este sentido, Dibelius sostenía que los textos sagrados debían leerse intentando recuperar su propósito inicial ligado al cristianismo primitivo y a lo que estos evangelios significaron para su generación, y no solo descubrir la relevancia de la figura de Pablo.

Más allá de las resonancias estrictamente teológicas que podemos encontrar en estas discusiones, lo que nos interesa resaltar son las preocupaciones por la temporalidad y por las concepciones de historia que se dirimen en los estudios exegéticos bíblicos, ya que las preguntas tienen un nudo común en la posibilidad de la actualización de las fuentes históricas y los usos del pasado.

Encontramos muchas veces, en numerosos intérpretes la descripción de la formación temprana de Hannah Arendt como simplemente ligada a la "teología y a la filosofía”, sin indagar en las fuentes ni en las líneas de investigación que guían a nuestra autora. Si bien, podemos

también porque nos ayuda a entender mejor la red de discusiones intelectuales que quiere marcar la historiadora y las posibles influencias de aquello en la formación temprana de Arendt. Lamentablemente no conseguimos el material.

11 “... Dibelius maintained that understanding the biblical figures and their message cannot be based on a psychological existentialist construction of their minds and personalities, but only on a historical-stylistic analysis of the "life and interests of the first Christian communities" (KEEDUS, 2015, p. 51). 
entender que Arendt por el vínculo con Heidegger habría estado en contacto con las mismas discusiones que su joven maestro, es necesario también preguntarnos qué conserva y que rechaza de la filosofía heideggeriana y, en general, de las interpretaciones de aquellas discusiones en esta obra de juventud. Entendemos que la disertación no puede leerse como un epígrafe a la filosofía heideggeriana, ya que aquí se expresan los primeros puntos de disidencia con su joven maestro. En esa línea, es menester retomar la tarea de articular este contexto de producción de la obra a los intereses que allí se expresan, en el horizonte de la pregunta por una posible continuidad de las indagaciones filosóficas que la joven autora abre en su tesis doctoral.

La reconstrucción del contexto intelectual nos sirve para dar cuenta de un entorno de crisis, de numerosas discusiones, donde encontramos a su vez la filosofía existencialista en ciernes. Esta pauta nos da elementos para pensar el germen de las ideas arendtianas de aquel período y en ese sentido, nos permiten corrernos de las lecturas escolares que atan a la pensadora alemana a sus maestros de juventud, resaltando solo a Jaspers y a Heidegger, sin considerar la efervescencia de las polémicas señaladas, a las cuales aludimos sucintamente.

\section{Rasgos existencialistas en el enfoque general de lectura}

Numerosos intérpretes han resaltado el vínculo de Arendt con Heidegger tomando en consideración la aproximación fenomenológica de su teoría política, sin embargo, prácticamente ninguno de ellos ha reparado en las fuentes de la formación temprana como mencionamos, pero es precisamente aquí donde Arendt, a partir de un lenguaje heideggeriano, comienza a su vez a distanciarse de la propuesta de su maestro. La lectura a contrapelo de Heidegger nos permite detectar los puntos filosóficos nodales de las afinidades y distancias con su maestro, para pensar en la apropiación de determinadas concepciones agustinianas en lugar de otras, en relación por ejemplo al problema de la temporalidad en un horizonte plural.

La generación de intelectuales de la que Heidegger era parte, y Arendt como estudiante comenzaba a tener contacto, compartió una serie de preocupaciones frente a la "objetualización" de las ciencias sociales en vistas a las consecuencias prácticas, políticas y éticas de las 
fuentes tradicionales de pensamiento. Muchos de aquellos intelectuales recurrieron a las fuentes teológicas y/o clásicas para ensayar otras respuestas a las pregustas que se gestaban a la luz de las acaloradas discusiones en el marco de los terribles acontecimientos de principio del siglo XX. Una de estas propuestas fue el retorno a las fuentes de la experiencia del cristianismo primitivo. En ese sentido, consideramos que la preocupación de nuestra autora y de Heidegger por Agustín se remite a esta recuperación en pos de la búsqueda de una experiencia que permita dislocar las categorías de la tradición, leerlas a la luz de otro contexto, y en este caso de un contexto de crisis: la caída del imperio Romano, a la luz de la experiencia cristiana y el descubrimiento del "hombre interior".

En su trayectoria como docente, Martin Heidegger, impartió numerosos seminarios que luego se cristalizaron en su gran obra de juventud, Ser y tiempo (1997); aunque suele considerarse el primer esbozo de los problemas trabajados en aquella obra el seminario titulado Ontología: hermenéutica de la facticidad (2000), dictado en 1923 en la Universidad de Friburgo. De todos modos, también podemos rastrear sus inquietudes y sus novedosos planteos en relación a la crítica a la metafísica tradicional en cursos anteriores. Como plantea Espósito, en el curso dictado en el semestre de invierno de 1921 en Friburgo sobre Agustín y el neoplatonismo (1999), el pensador alemán señala en el hiponense determinada antropología filosófica y la analiza a partir del planteo del problema del ser-ahí histórico-temporal. La presencia de Agustín de Hipona en el pensamiento heideggeriano se "traducirá luego como un intento por "metabolizar" una tendencia fundamental agustiniana, aunque oculta, en su propio pensamiento" (ESPÓSITO, 2009, p. 433). Al inicio del artículo Espósito menciona que:

Dos son las principales tendencias de la interpretación heideggeriana de Agustín: 1) una fenomenología del yo, entendida como el modo en el cual la vida factual se comprende a sí misma, y con esto se cumple como existencia o "ser ahí" (Dasein), y 2) una interpretación de este ser ahí como tiempo, entendido - o mejor, vivido, y entonces cumplido- como la apertura al misterio del sentido del ser. Es propiamente este "cumplimiento" (Vollzug) el signo, pero también el aporte fundamental de la presencia de Agustín en el pensamiento de Heidegger: pero no en el sentido de que en él el filósofo alemán llegue a encontrar respuesta a los propios interrogantes, sino en el sentido de que de él recoge la interrogación como el modo de ser fundamental de la vida, pero también —con Agustín, contra Agustín - resuelve la vida en la imposibilidad de dar respuesta a aquella interrogación, y entonces la cumple en absoluta finitud (ESPÓSITO, 2009, p. 434). 
Por este motivo, sigue Espósito, como menciona al inicio del seminario sobre Agustín y el neoplatonismo (1999), Heidegger tomará distancia de las "modalidades de aproximación" a la lectura de Agustín en boga en ese momento. Heidegger comentará de manera crítica pero sucintamente tres representantes de aquellas: Troeltsch, y su "historia cultural"; Von Harnack, y su "historia de los dogmas"; y Dilthey y su "ciencia histórica". Aquellas aproximaciones, suponían leer a Agustín como un objektgeschichtlich, es decir como un objeto histórico, "cuyo foco problemático estaría constituido por la relación entre lo griego y el cristianismo o, dicho al revés, por el proceso de "helenización del cristianismo" (ESPÓSITO, 2009, p. 434). Al contrario, la interpretación heideggeriana proponía leer a Agustín desde una aproximación "histórico-ejecutiva" (vollzugsgeschichtlich). El pensador alemán encuentra en la obra agustiniana una experiencia radical, en la cual la historia es leída como "historicidad" en el sentido del "cumplimiento" ontológico de la existenz: "este cumplimiento existencial es comprendido "históricamente" como relación problemática - irresuelta y en definitiva irresoluble- del yo consigo mismo" (ESPÓSITO, 2009, p. 435). Esto es lo que más tarde Arendt llamará “el descubrimiento del hombre interior" (ARENDT, 2005, p. 42; 2018, p. 287-316) de la filosofía cristiana y medieval en general y en particular de la de Agustín.

Tanto para Heidegger como para Arendt, Agustín es el gran pensador cristiano que "produce la primera hermenéutica de gran estilo" (HEIDEGGER, 2000, p. 30), articulando una pregunta que "toda filosofía anterior había dado por supuesta": "Lo que san Agustín espera de Dios es una respuesta a la cuestión “iquién soy yo?” (ARENDT, 2001, p. 43). El tratamiento de la quaestio12 agustiniana, "me he convertido en una cuestión para mí mismo" (AGUSTÍN, 2005, X,

\footnotetext{
${ }^{12} \mathrm{Al}$ inicio de Ontología: Hermenéutica de la facticidad Heidegger retoma la distinción entre los "problemas" y las "cuestiones", lo cual nos daría una pauta de lectura. Allí menciona que: "Cuestiones surgen sólo del habérselas con las "cosas" [...].Así pues, hay que "plantear" aquí algunas cuestiones, tanto más cuanto que el cuestionar ha caído en desuso con el gran negocio de los "problemas". Aún más, se pretende de manera solapada acabar definitivamente con el cuestionar, pensando en alimentarse la simplicidad de la fe del carbonero. Se proclama que lo sacrum es la ley de la esencia, y la época, que por su fragilidad y falta de enjundia está necesitada de ello, se lo toma en serio"(HEIDEGGER, 2000, p. 21).
} 
$33,50)^{13}$, implica una "mirada retrospectiva sobre la propia vida [que] no está al servicio, en cambio, de la propia fama, sino de la gloria de Dios" (ARENDT, 2005, p. 42).

En el curso sobre Hermenéutica de la facticidad, Heidegger, menciona que la labor de aquella "disciplina" es:

...hacer el existir propio de cada momento accesible en su carácter de ser al existir mismo, de comunicárselo, de tratar de aclarar esa alienación de sí mismo de que está afectado el existir. En la hermenéutica se configura para el existir una posibilidad de llegar a entenderse y de ser ese entender (HEIDEGGER, 2000, p. 33).

Es significativo el hecho de que desde la perspectiva hermenéutica agustiniana, según la lectura de Heidegger, el punto de partida sea la experiencia de la fe para poder escuchar el verbum Dei; esto es, el crede ut intelligas ${ }^{14}$, que se traduce como un retorno a sí mismx, a la vida singular de unx; solo aquel fundamento, que es considerado como la experiencia más profunda y plena de sí, sirve de base del conocimiento (ESPÓSITO, 2009, p. 438):

Es este el auténtico descubrimiento agustiniano: un descubrimiento decisivo, gracias al cual se manifiesta para Heidegger "un aspecto absolutamente originario" de la existencia: "Agustín ve en el inquietum est cor nostrum la gran incesante inquietud de la vida” (ESPÓSITO, 2009, p. 38).

Es aquí donde Espósito identifica uno de los puntos nodales de vinculación entre Heidegger y Agustín, es decir, la conexión entre "la inquietud de la existencia y la pregunta por el sentido del ser", la cual nos provee de un indicio del recorrido heideggeriano propuesto a partir

\footnotetext{
${ }^{13}$ Ninguna traducción al castellano de las Confesiones traduce la frase "quaestio mihi factus sum" tal como lo traduzco aquí; la edición de la BAC lo traduce como "me he convertido en un problema para mí mismo" y la traducción de la Dra. Magnavacca como "me he convertido en un problema". Entendemos que si bien a fines pedagógicos es preferible la traducción de quaestio como problema, consideramos que en español contamos con la palabra "cuestión" que es mucho más literal, amplia y neutral. Por otro lado, ver la nota 12 del presente artículo. A veces la traducción de la BAC, hay muchos acentos que deja de lado que permitirían una aproximación más fina al texto, que en cambio Silvia Magnavacca sabe resaltar. Un ejemplo de esto es la palabra latina quaestio.
}

${ }^{14}$ Ver nota 12, de ESPÓSITO, 2009, p. 433-462. 
del descubrimiento de la facticidad del cristianismo primitivo de Agustín. La experiencia protocristiana indica un punto de inflexión respecto de las vertientes que identificaban la "inteligencia" con la objetivación teorética ${ }^{15}$.

La experiencia de la inquietud de sí en su articulación constitutiva con la "cura" o "cuidado" le proporcionó al maestro de Maburgo los elementos para detectar la tendencia ontológica decisiva de nuestro ser-en-el mundo. El problema que detecta aquí Espósito es que, a la luz de la Destruktion heideggeriana, para Heidegger la tendencia fundamental que se desprende de la experiencia greco-cristiana de Agustín es la necesidad de la auto-comprensión ${ }^{16}$, donde la aperturidad (sich erschlieBen) constitutiva del Dasein como condición de comprensión de sí, del mundo y de los otros (mis congéneres), al final resulta la posibilidad o imposibilidad de la autocomprensión misma.

En el artículo Agustín de Hipona y el protestantismo, Arendt menciona que:

En el instante de la conversión, Agustín es salvado por Dios; no lo es el mundo todo, sino él, el individuo, el que está ante Dios; él es salvado de su vida en pecado. Y el hecho de que él confiese esta salvación sucede para gloria de Dios, y es testimonio humano del poder de Dios. Tal confesión le obliga a recordar su vida pasada, y en realidad toda su vida, pues cada momento de ella era pecado, de modo que cada momento acrece la magnitud y el milagro de la salvación. Con ello la propia vida

${ }^{15}$ En este punto, Espósito señala la articulación entre aquella tendencia protocristiana agustiniana y el redescubrimiento de la "física" aristotélica de la vida. Justamente sobre este vínculo Heidegger piensa la existencia del Dasein como cura o cuidado (Sorge), "gracias a una interpretación de la antropología agustiniana a la luz de la ontología aristotélica" (ESPÓSITO, 2009, p. 439), tal como reza en la nota 3 del parágrafo 42 de Ser y tiempo: "El enfoque del "cuidado" que ha orientado la precedente analítica existencial del Dasein, se le fue aclarando al autor a través de los intentos de una interpretación de la antropología augustiniana -es decir, greco-cristiana- en relación a los principios fundamentales de la ontología de Aristóteles" (HEIDEGGER, 1997, p. 220, itálicas nuestras). La recuperación de la experiencia cristiana agustiniana se vincula con su crítica a la tradición, descubriéndola como una historia de encubrimientos, que ha escondido siempre el originario ser- histórico, como menciona el intérprete.

${ }^{16}$ Lo que dice aquí el intérprete es que según la lectura heideggeriana la inquietud de sí puede decantar en la autocomprensión (como posibilidad), mediada por la estructura de la cura. Si bien, el problema de la autocomprensión en Agustín "está bien testimoniada" el horizonte nunca es sólo el sí mismo. Ver Agustín de Hipona. (2005) Confesiones, X, 5, 7: "Tú eres, Señor, quien me juzga. Porque, aun cuando nadie entre los hombres sabe las cosas de un hombre sino el espíritu que está en él, hay, con todo, algo que ni siquiera conoce el espíritu que está en él...Confesaré, pues, lo que de mí sé; confesaré también lo que de mí ignoro, porque también lo que sé de mí, lo sé porque me iluminas; lo que de mí ignoro, no lo sabré hasta que mis tinieblas se hagan como mediodía ante tu rostro". 
se vuelve una trama unitaria llena de sentido, un camino de salvación (ARENDT, 2005a, p. 42-43, itálicas nuestras).

El aspecto aquí señalado remite a la pregunta por el sentido de la propia vida, como una unidad de sentido, es decir, en singular, aunque a la par de la recuperación de la "realidad salvada". El recuerdo, dice Arendt, permite abrir este camino de salvación. Es a propósito del tratamiento de la memoria agustiniana donde los caminos de la lectura arendtiana y heideggeriana de Agustín se bifurcan: mientas para Heidegger, la memoria tiene una relevancia nodal en el marco de la "aporía del olvido" (oblivio) que nos remite la búsqueda de sí, que debe ser rebasada para el encuentro con Dios ${ }^{17}$; para Arendt, la memoria me expone y expone los prejuicios de la selección de lo recordado, por eso lo ubica como un "recuerdo falseador" (ARENDT, 2005a, p. 43). Este recuerdo falseador da con la interioridad del cristiano, la cual, sin embargo no se concibe como autónoma ni tiene como horizonte de búsqueda la conciencia individual. La pregunta por la propia interioridad aquí pierde sentido fuera de un horizonte plural:

Pues la interioridad no tiene valor por el hecho de ser la mía y ser por ello interesante, sino que lo tiene porque era mala y ha llegado a ser buena. La propia vida no merecerá atención por ser individual y única en un sentido moderno, ni por tener su propio desarrollo en el sentido de un proceso de perfeccionamiento de la personalidad: no es que la propia vida sea única, es que es ejemplar; como a mí me ha ido, así les va a todos. La confesión personal tiene sentido general: así es como impera la gracia de Dios en una vida en general. La vida no tiene una historia autónoma, sino que el principio fundamental de cambio es la conversión, que divide la vida en dos mitades (ARENDT, 2005a, p. 43).

Aquí, sigue Arendt, se abren dos interpretaciones de la actitud confesional frente al recuerdo: por un lado como confesión sacramental, por otro, como conciencia moral (individual). Para la primera el confitieri es arrojado a la propia interioridad, que en el proceso de confesión

\footnotetext{
17 "Mi vida es memoria, y entonces yo mismo soy olvido. Es precisamente aquí donde nace, o más bien renace desde el fondo la pregunta, pero sería mejor decir la invocación de Agustín: Quid ergo sum, Deus meus? (AUGUSTíN, 2005, p. X, 17, 26), que Heidegger traduce en el más neutro interrogativo fenomenológico: “iqué significa buscar?” (Was heißt Suchen?)" (ESPÓSITO, 2009, p. 441). El problema con la memoria agustiniana según Heidegger es que el pensador alemán hace decantar de aquella la angustia constitutiva del miedo a la muerte, sin considerar importar el egoísmo del ente que pregunta, que se preocupa por la salvación individual. Según la traducción Jacobo Muñoz de Agustín y el neoplatonismo, allí Heidegger tiene presente aquella tendencia egoísta; no ya en Ser y tiempo. Claramente este tema merece un tratamiento más minucioso de lo que aquí proporcionaremos por cuestiones de tiempo y espacio.
} 
se expone ante Dios, y de él a los otros, para servir de ejemplo. Esta mediación reflexiva ante Dios, fue combatida fuertemente por Lutero, quien defendió "la concepción del creyente como el hombre cuya conciencia moral está sin mediaciones ante Dios" (ARENDT, 2005a, p. 44). De este modo, "se tornó meditación reflexiva sobre la propia vida sin más".

Es en la vertiente luterana de las lecturas de Agustín donde ubicamos a la interpretación heideggeriana, la cual pone de relieve la vuelta a la interioridad por la salvación individual de cara a la gracia de Dios. Aquí no nos abocaremos a realizar una lectura sistemática de su sinuoso artículo sobre la Filosofía de la existencia, porque excede nuestro trabajo (aún si tomamos solo el apartado sobre Heidegger), pero lo que queremos resaltar es la continuación de aquella crítica que se expresa en el pequeño artículo de 1930 y más tarde en ¿Qué es filosofía de la existencia? (ARENDT, 2005c) En palabras de Di Pego:

Las críticas que allí [en ¿Qué es Filosofía de la Existencia?] esgrime a Heidegger deben concebirse como una profundización de las formulaciones tempranas en su libro sobre Agustín. De manera que puede apreciarse una línea de continuidad entre las dos primeras etapas de la relación intelectual con su maestro que, luego de una muy breve fascinación inicial, da lugar a un camino de paulatino fortalecimiento de los primeros bosquejos críticos (DI PEGO, 2019, p. 204).

\section{Arendt con, más allá de la tradición}

Como menciona Porcel, el término "tradición” es utilizado por Arendt de manera inequívoca a lo largo de su obra. Sin embargo, y en articulación con su análisis y diagnóstico de la modernidad, todas las aserciones en relación a ella comparten un punto de apoyo común: el reconocimiento - a fin de comprender - del quiebre de la tradición política de pensamiento que vio gestar los acontecimientos catastróficos del siglo XX (PORCEL, 2016, p. 212).

La ruptura radical del "hilo rojo de la tradición”, como dirá Arendt en escritos posteriores, fue anunciada por numerosos pensadores a principios del siglo XX, como vimos. El quiebre de la tradición respondía a su incapacidad de retomar las respuestas que la misma tradición se había dado a sí misma para explicar determinados acontecimientos; ella era incapaz de articular la experiencia histórica reciente con las categorías filosóficas, políticas y epistemológicas 
disponibles. Así, una generación de intelectuales intentó responder a esta imposibilidad, primero denunciando esta incapacidad, y luego ensayando posibles nuevas respuestas, dando lugar a otras formas de afrontar las preguntas que surgían en ese contexto. Aquellos intelectuales discurrieron en numerosas y acaloradas discusiones en torno a la temporalidad y la historia. Los discípulos de Gogarten, como mencionamos, se definieron a sí mismos como la generación between the times, aludiendo a los planteos de su maestro en relación a la no-pertenencia a "ninguna época": ya no pertenecemos al tiempo presente que está llegando a su fin; pero aún no pertenecemos al periodo histórico por-venir.

El horizonte intelectual abierto por y para aquella generación, que se había topado con esta ruptura, nos remitía a la experiencia de un abismo temporal. Como Arendt escribe posteriormente en su ensayo sobre La muerte de Virgilio:

Pues el declinar de lo antiguo y el nacimiento de lo nuevo no es necesariamente una cuestión de continuidad; entre las generaciones, entre quienes por una u otra razón aún pertenecen a lo antiguo y quienes en su propia piel sienten llegar la catástrofe o han crecido ya con ella, la cadena está rota y un "espacio vacío" emerge, una suerte de tierra de nadie en términos históricos, que sólo puede describirse con las palabras "ya no y todavía no". Una tal interrupción absoluta de la continuidad tuvo lugar en Europa durante y después de la Primera Guerra Mundial (ARENDT, 2005b, p. 197).

Aquel horizonte intelectual de ruptura y de la experiencia de un abismo -en términos históricos primero, en términos teóricos, después-, podía describirse a partir de dos aspectos: uno negativo, que remarcaba la incapacidad de las categorías disponibles dadas por la tradición para explicar semejantes acontecimientos; uno positivo, que exponía la necesidad de crear un horizonte de comprensión novedoso frente a esta tradición de pensamiento avejentada e incapaz de responder a su panorama histórico. Este "pathos de la novedad" no significó siempre una propuesta positiva, aunque, hubo algunos intentos por definir estas novedosas perspectivas de investigación, que en principio necesitaban romper con los viejos enfoques basados en la subjetividad y en la percepción del mundo a partir del sujeto, por un lado, y en las líneas metodológico- epistemológicas formalistas, principalmente ligados al neokantismo, por otro (KEEDUS, 2015, p. 63). 
En Arendt esta preocupación se articuló con la problematización de la relación entre tradición de pensamiento y condiciones de posibilidad del mundo común. La cita de René Char, que en numerosas ocasiones nuestra autora trae, "Nuestra herencia no está precedida por ningún testamento", nos da una pauta del tipo de ruptura que piensa. Si bien, hay una ruptura radical que implica una incapacidad de tomar las categorías disponibles sin problematizarlas, no se trata de desechar todos los esfuerzos intelectuales previos para desandar tales caminos, sino de rechazar a la tradición como un "todo", como una unidad de sentido. Aunque, en términos de Char, no haya testamentos, es decir pautas y respuestas fijadas por una tradición de pensamiento, somos herederos de un pasado y de determinadas experiencias históricas. Se trata más bien de un esfuerzo que convoca a la tradición para pensar más allá de ella, en un panorama que nos permita su crítica y su actualización en un nuevo horizonte histórico-filosófico.

Porcel lo formula del siguiente modo:

Arendt establece -a partir de su convicción de la ruptura del hilo de la tradición- que existe la posibilidad de pensar y de actuar sin la guía de la tradición y de entablar un vínculo nuevo con un pasado que ha escapado a la autoridad del pensamiento heredado (PORCEL, 2016, p. 213).

Las fuentes del pasado son recuperadas y puestas a la luz de otra propuesta dialógica con el tiempo y la historia. Tal como lo expresó el joven Heidegger en 1921, en el seminario impartido en Friburgo sobre Agustín ${ }^{18}$ :

La historia nos afecta, y nosotros somos ella misma; y precisamente por no ver esto, cuando creemos poseer y dominar una consideración objetiva de la historia hasta hoy nunca alcanzada, precisamente porque pensamos esto y en esta opinión seguimos imaginando y construyendo presunta cultura y filosofía y sistemas, hora tras hora nos golpea con mayor fuerza la historia a nosotros mismos. El discurso sobre el "estar en las dimensiones efectuales" no tiene nada que ver con el lugar común de que siempre se es dependiente de la tradición (HEIDEGGER, 1999, p. 25).

\footnotetext{
${ }^{18}$ Este curso se titula Agustín y el neoplatonismo. En la primera parte lo que hace Heidegger es intentar aclarar el modo de abordaje que propone de la obra de Agustín, alejándose explícitamente de tres posiciones y modos de entablar relaciones con la historia, estos personajes son Troeltsch, Dilthey y von Harnak. Allí plantea el "problema de la objetividad histórica" y frente a ella comienza a dibujar un acercamiento en un sentido existencial, como mencionamos.
} 
Las “dimensiones efectuales" hacen referencia al sentido de ejecución como el “cómo del experimentar" específico. En las primeras páginas Heidegger delinea su propuesta de lectura, alejándose como reza la cita de las perspectivas que presuponen una consideración objetiva de la historia. Con todo, no hay que caer en lecturas precipitadas y plantear que lo "contrario" a lo histórico- objetivo es "subjetivo" (HEIDEGGER, 1999, p. 22). La lectura que plantea Heidegger de Agustín intenta dar lugar a "nexos de sentido", sacando a la luz ciertos fenómenos decisivos, que se determinaron como decisivos en la época patrística, cuándo vivió Agustín, y "siguen todavía “sustentándonos" a nosotros" (HEIDEGGER, 1999, p. 24).

Sin embargo, el punto distintivo heideggeriano lo encontramos en las primeras aserciones acerca de determinados conceptos que luego funcionarán como pilares en el marco de la “analítica existencial”, y encontrarán plena articulación en obras posteriores.

Como vimos, el historicismo, una de las líneas hegemónicas de la modernidad filosófica, detentó una serie de consecuencias prácticas, así como también una forma particular de relacionarse con el pasado y sus fuentes. En ese sentido, muchos de sus críticos -uno de ellos, Heidegger- intentaron entablar otro tipo de relación con el pasado tomado como fuentes filosóficas las anteriores a las que formuló la modernidad; muchas veces esto se hizo a partir de una recuperación de las filosofías clásicas o determinadas líneas de las filosofías medievales. Volver a estas fuentes implicaba un esfuerzo por tender puentes que permitan leer esta brecha temporal como tal. La recuperación de San Agustín por Arendt puede comprenderse como un esfuerzo en consonancia con esta lectura. Retomando a Bodei diremos que quizás reparar en el pensamiento de Agustín aún nos permita "proyectar luz indirecta también sobre la naturaleza de los obstáculos con que ha tropezado la modernidad y de los nudos que todavía quedan eventualmente por desatar" (BODEI, 1998, p. 154).

Como mencionan las editoras de la versión en inglés de El concepto de amor en San Agustín, la publicación de esta nueva traducción intenta estimular nuevas conversaciones acerca de la relación de Arendt con la "tradición filosófica" (SCOTT \& STARK, 1996, p. 116). El diálogo crítico con la tradición que nuestra autora teje a lo largo de su obra puede vincularse en ese sentido con sus primeros acercamientos a Agustín en el marco de su formación temprana. De 
todas maneras, en su tesis doctoral no encontramos más que una crítica que se encuentra en ciernes y que en el marco de toda la obra de Arendt debe rastrearse tomando en consideración un sinuoso camino de diálogos complejos.

\section{Referencias bibliográficas}

ARENDT, H. Martin Heidegger at Eighty. In: MURRAY, M. (Ed.). Heidegger and modern philosophy. Critical essays. 2da edición. United States of America: Yale University, 1978.

AREND, H. El concepto de amor en San Agustín. España: Encuentro, 2001.

ARENDT, H. Agustín y el protestantismo. In: Ensayos de comprensión 1930-1954. Madrid: Caparrós editores, 2005a.

ARENDT, H. Ya no, todavía no. In: Ensayos de comprensión 1930-1954. Madrid: Caparrós editores, 2005b.

ARENDT, H. ¿Qué es la filosofía de la existencia? In: Ensayos de comprensión 1930-1954. Madrid: Caparrós editores, 2005c.

ARENDT, H. La tradición y la época moderna. In: Entre el pasado y el futuro: ocho ejercicios sobre la reflexión política. Buenos Aires: Ariel, 2016a. p. 33-66.

ARENDT, H. La condición humana. Barcelona: Paidós, 2016b.

ARENDT, H. Quaestio mihi factus sum: el descubrimiento del hombre interior. In: La vida del espíritu. Barcelona: Paidós básica, 2018. P. 287- 380.

AUGUSTÍN. Confesiones. Buenos Aires: Losada, 2005.

AUGUSTÍN. La ciudad de Dios. Madrid: Biblioteca de Autores Cristianos (BAC), 1958.

BARTH, K., The epistle to the romans. 6ta edición. London: Oxford University Press, 1933.

BENHABIB, S. The reluctant modernism of Hannah Arendt. 2 da edición. United States of America: Rowman \& Littlefield Publishers, 2003.

BODEI, R. Ordo amoris: conflictos terrenos y felicidad terrestre. 2da edición. Madrid: Cuatro ediciones, 1998.

CANOVAN, M. A reinterpretation of political thought. New York: Cambridge University Press, 2002.

DI PEGO, A. En torno al pensamiento: la disputa de Hannah Arendt con Martin Heidegger Tópicos, México, núm. 56, 2019. 197-235. Recuperado de: https://www.redalyc.org/journal/3230/323062667008/html/

ESPÓSITO, C. La memoria, la tentación y el tiempo. Revista De Investigación E Información Filosófica, 65(245), 2009. 433-462. Recuperado de: https://revistas.comillas.edu/index.php/pensamiento/article/view/2716.

FORTI, S. La vida del espíritu y el tiempo de la polis. Madrid: Ediciones Cátedra, 2001.

HEIDEGGER, M. Ser y tiempo. Santiago de chile: Editorial Universitaria, 1997.

HEIDEGGER, M. Ontología: hermenéutica de la facticidad. Madrid: Alianza editorial, 2000.

HEIDEGGER, M. Agustín y el neoplatonismo. In: Estudios sobre mística medieval. 2da edición. México DF: Fondo de cultura económico, 1999. P. 13- 158. 
KEEDUS, L. The crisis of the German historicism: the early political thought of Hannah Arendt and Leo Strauss. United Kingdom: Cambridge University Press, 2015.

PORCEL, B. Tradición. In: PORCEL, B. \& MARTÍN, L. (Comps.) Vocabulario Arendt. Rosario: Homo Sapiens Ediciones, 2016. P. 211- 221.

SCOTT, J. V. \& STARK, J. C. Rediscovering Hannah Arendt. In: ARENDT, H. Love and St. Augustine. Unite States of America: University of Chicago Press, 1996. P. 115- 212.

YOUNG- BRUEHL, E. Hannah Arendt. Una biografía. 2da edición. Barcelona: Paidós Ibérica, 2006.

Recebido em: 30/8/2021

Aprovado em: 06/10/2021

\section{Ari Angelina Costamagna Fernández}

Es egresada del Profesorado en Filosofía de la Universidad Nacional de Córdoba (Argentina) y profesora adscripta de las cátedras Filosofía Contemporánea y Ética II en la misma casa de estudios. Además, es investigadora del Centro de Investigaciones María Saleme de Burnichón (CIFFYH-UNC), donde participa del grupo "El retorno de lo común: filosofía, crítica y práctica política", a cargo del Dr. Sebastián Torres y la Dra. Paula Hunziker. Actualmente es tesista, en cuya investigación desarrolla la lectura arendtiana de Agustín de Hipona a partir de la obra temprana, centrada en El concepto de amor en San Agustín, y las líneas problemáticas de continuidad con la obra posterior. Fue becaria del Consejo Interuniversitario Nacional (CIN) con la investigación titulada "Temporalidad de la política e institución-constitución de lo común: el problema de la promesa en la teoría política de Hannah Arendt". 